INRA Prod. Anim., 2005, 18 (3), 159-168

\title{
Variabilité inter et intra matière première de la teneur en phosphore total et phytique et de l'activité phytasique
}

G. TRAN 1 , F. SKIBA ${ }^{2}$

1 io - la Banque de Données de l’Alimentation Animale, Association Française de Zootechnie, F-75231 Paris Cedex 05

2 Arvalis - Institut du végétal, Pouline, F-41100 Villerable

Courriel :tran@inapg.fr

La maîtrise des apports nutritionnels de phosphore et le contrôle des rejets dans l'environnement nécessitent une bonne connaissance des quantités de phosphore ingérées par l'animal via l'aliment et les matières premières qui le composent. La valeur «phosphore» des aliments est caractérisée par trois critères : la teneur en phosphore total, la teneur en phosphore phytique et l'activité phytasique endogène de la matière première.

Sauf mention contraire, les données présentées dans cet article proviennent de io - la Banque de Données de l'Alimentation Animale.

\section{1 / Teneurs en phosphore total}

\section{1 / Aspects généraux}

Compte tenu de la grande diversité des matières premières, leurs teneurs en phosphore total varient très largement :

Tableau 1. Teneurs en phosphore total (moyenne et écart-type), ratio phosphore phytique sur phosphore total et activité phytasique de quelques matières premières (INRA-AFZ 2004 et Skiba, données non publiées).

\begin{tabular}{|l|c|c|c|}
\hline \multicolumn{1}{|c|}{ Nom } & $\mathbf{P}(\mathbf{g} / \mathbf{k g}$ brut) & $\begin{array}{c}\text { P phytique / P total } \\
(\%)\end{array}$ & Activité phytasique (U/kg) \\
\hline Avoine & $3,2(0,4)$ & 55 & 40 \\
\hline Blé tendre & $3,2(0,3)$ & 65 & 20 \\
\hline Maïs & $2,6(0,3)$ & 75 & 538 \\
\hline Orge & $3,4(0,3)$ & 55 & 770 \\
\hline Seigle & $3(0,3)$ & 65 & 3080 \\
\hline Triticale & $3,5(0,4)$ & 65 & 2590 \\
\hline Farine basse de blé tendre & 3,6 & 80 & 1770 \\
\hline Remoulage demi-blanc de blé tendre & $8,7(1,4)$ & 80 & 0 \\
\hline Son de blé tendre & $9,9(1,1)$ & 80 & 120 \\
\hline Corn gluten feed & $8,9(1,5)$ & 65 & 0 \\
\hline Son de riz gras & $16,1(2,1)$ & 85 & 50 \\
\hline Graine de colza & $6,6(0,9)$ & 70 & 140 \\
\hline Féverole à fleurs blanches & $4,7(0,3)$ & 60 & 130 \\
\hline Lupin blanc & $3,8(0,7)$ & 60 & 0 \\
\hline Pois & $4,0(0,5)$ & 45 & 0 \\
\hline Graine de soja extrudée & $5,5(0,5)$ & 60 & 10 \\
\hline Graine de tournesol & $5,4(0,9)$ & 85 & 20 \\
\hline Tourteau de colza & $11,4(0,9)$ & 60 & 0 \\
\hline Tourteau de soja 48 & $6,2(0,5)$ & 60 & 0 \\
\hline Tourteau de tournesol non décortiqué & $10,1(1,4)$ & 85 & 0 \\
\hline Manioc, amidon 67\% sur brut & $0,9(0,3)$ & 25 & 0 \\
\hline Mélasse de canne & $0,6(0,3)$ & 10 & 0 \\
\hline Pulpe d'agrumes déshydratée & $0,9(0,2)$ & 40 & 0 \\
\hline Pulpe de betterave déshydratée & $0,9(0,3)$ & 10 & 0 \\
\hline Luzerne déshydratée, protéines $17-18 \%$ sur sec & $2,4(0,3)$ & 5 & 0 \\
\hline Paille de blé & $0,7(0,3)$ & 5 & 0 \\
\hline Lactosérum acide déshydraté & 10,1 & 0 & 0 \\
\hline Farine de poisson type 65 & $25,2(3,0)$ & 0 & \\
\hline
\end{tabular}


Figure 1. Teneurs en phosphore total ( $\mathrm{g} / \mathrm{kg}$ brut) par famille de matières premières non minérales (INRA-AFZ 2004).

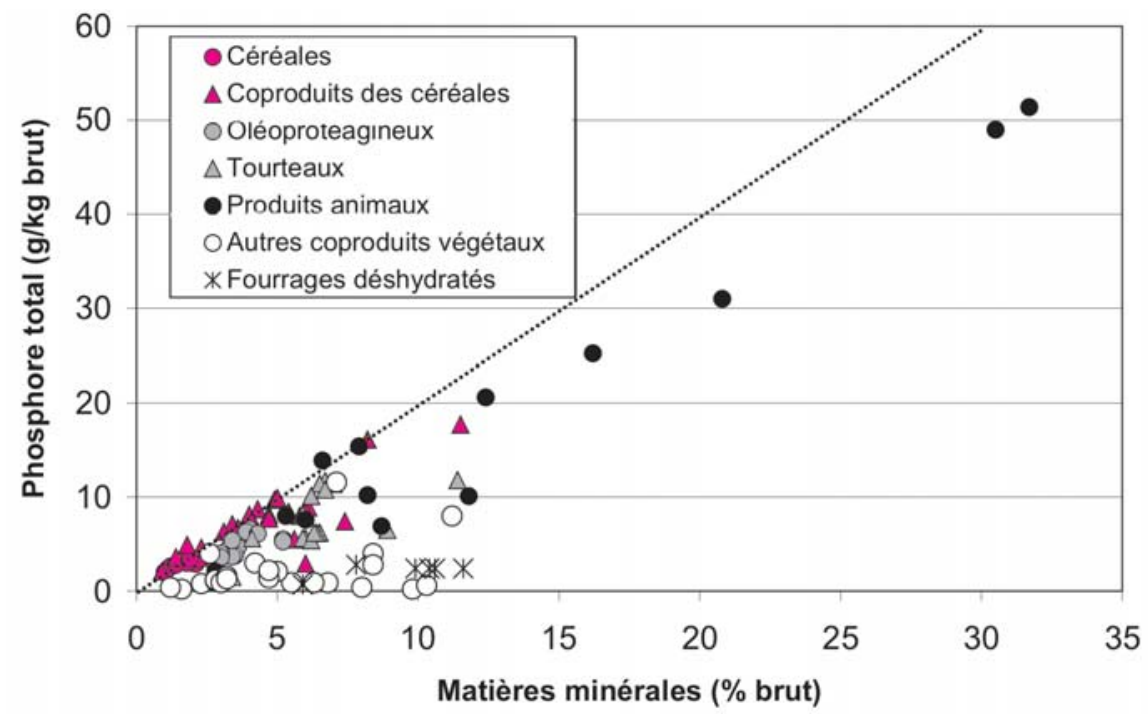

de moins de $1 \mathrm{~g} / \mathrm{kg}$ pour des produits tels que la paille de blé à plus de $210 \mathrm{~g} / \mathrm{kg}$ pour certains phosphates. Dans le cas des produits non minéraux (figure 1), les matières premières les plus riches sont les coproduits animaux contenant des os (farines de viande, interdites dans l'Union Européenne) ou des arêtes (farines de poisson). Les produits végétaux les plus riches sont les sons de riz et certains tourteaux (sésame, coton, colza, tournesol) (figure 2).

Le phosphore représente généralement moins de 20 \% des matières minérales totales. Lorsque l'on compare les différentes familles de matières premières, il n'existe pas de relation globale entre la teneur en phosphore total et la teneur en matières minérales. Par contre, de telles corrélations apparaissent au sein des différentes familles bota-

niques. La nature et la qualité de ces corrélations dépendent fortement de la nature biologique des produits. Elles sont surtout pertinentes pour les grains, graines et leurs coproduits, ainsi que pour les produits animaux.

Pour d'autres familles de matières premières, telles que les fourrages frais ou déshydratés et certains coproduits industriels (pulpes de betteraves, pulpes d'agrumes), les teneurs en phosphore des matières premières sont faibles à la fois en valeur absolue et en valeur relative par rapport aux matières minérales. Elles sont aussi peu corrélées aux teneurs en matières minérales.

\section{2 / Céréales et leurs coproduits}

Les grains de céréales contiennent de 2 à 4 g/kg sur produit brut de phospho-

Figure 2. Variation par famille de matière première (famille botanique pour les végétaux) de la teneur en phosphore total ( $\mathrm{g} / \mathrm{kg}$ brut) pour les produits ayant moins de $8 \%$ de matières minérales.

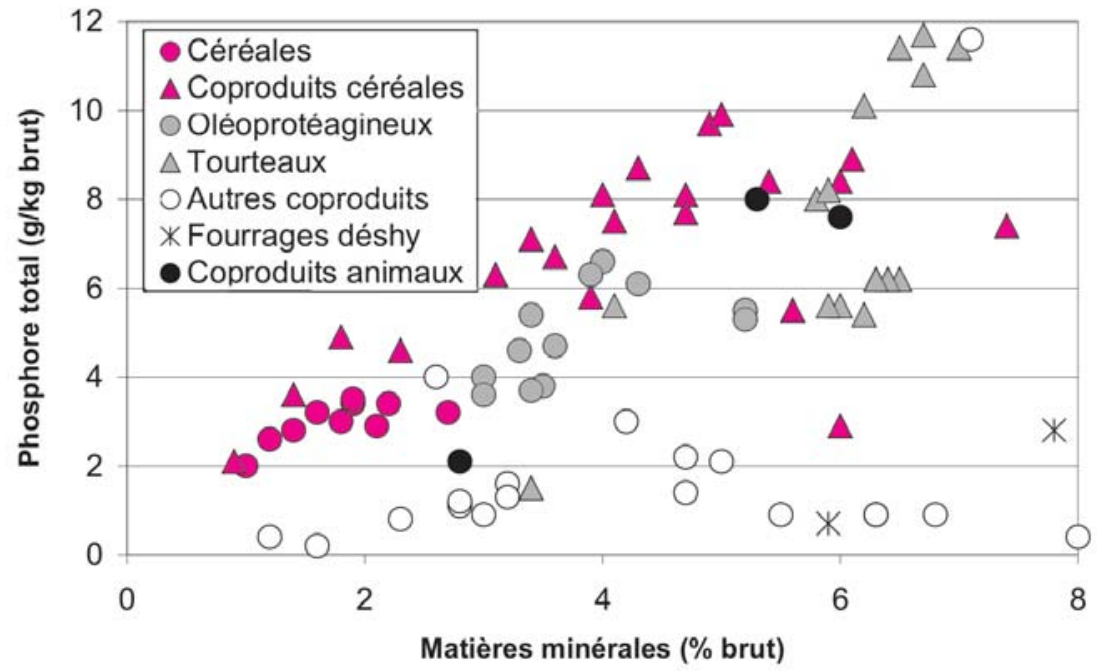

re total. Le maïs est légèrement moins riche $(2,6 \mathrm{~g} / \mathrm{kg})$ que les autres grains (3-3,4 g/kg), mais il contient aussi moins de matières minérales. L'écart type pour les grains de céréales est de l'ordre de 0,35 g/kg (CV de $10 \%$ ). Le blé et maïs ont les ratios phosphore /matières minérales ( $\mathrm{P} / \mathrm{MM})$ les plus élevés des matières premières non minérales (20-22 \% des matières minérales) (figure $3 a$ ).

Dans le cas du blé et des issues de meunerie (figure $3 \mathrm{~b}$ ), il existe une continuité dans la relation reliant phosphore et matières minérales entre le grain d'origine et les coproduits : $10 \mathrm{~g} / \mathrm{kg}$ de matière minérale contiennent environ $2 \mathrm{~g} / \mathrm{kg}$ de phosphore total. Le fait d'extraire l'amidon entraîne une concentration en matières minérales qui se traduit par une concentration en phosphore.

\section{3 / Graines oléo protéagineu- ses et tourteaux}

Les graines oléagineuses et protéagineuses sont plus riches en matières minérales et en phosphore total que les céréales : leur teneur en $\mathrm{P}$ total est comprise entre 2,5 et $8,5 \mathrm{~g} / \mathrm{kg}$ sur produit brut avec un écart-type intra matière première de l'ordre de 0,6 et un CV voisin de $13 \%$. Le pois et le lupin ont des teneurs en phosphore comparables $(3,8-4 \mathrm{~g} / \mathrm{kg})$ et inférieures à la féverole $(4,6 \mathrm{~g} / \mathrm{kg})$. Les ratios $\mathrm{P} / \mathrm{MM}$ de ces trois protéagineux sont en revanche plus faibles que pour les céréales (13\% pour le pois et la féverole, $11 \%$ pour le lupin).

Les graines de tournesol, colza et soja diffèrent notablement les unes des autres (figure 4) : le colza est le plus riche en phosphore, tant en valeur absolue $(6,6 \mathrm{~g} / \mathrm{kg}$ avec un écart type de 0,9 soit un $\mathrm{CV}$ de $14 \%$ ) qu'en valeur relative (16,5 \% des matières minérales). La graine de soja et le tournesol présentent des valeurs absolues comparables (de l'ordre de $5,4 \mathrm{~g} / \mathrm{kg}$ ) mais le soja a un ratio $\mathrm{P} / \mathrm{MM}$ relativement faible, de l'ordre de $10 \%$.

Comme dans le cas des issues de blé, il existe une continuité entre la graine et les coproduits correspondants (figure 5). Dans le cas des graines oléagineuses, on retrouve au niveau des tourteaux les différences observées sur les graines. Les tourteaux de tournesol ont une variabilité importante de leur composition chimique globale, qui se répercute sur la teneur en phosphore. Alors que les tourteaux de colza et de tournesol ont des teneurs moyennes en 
Figure 3a. Teneurs en phosphore total ( $g / k g$ brut) des principales céréales.

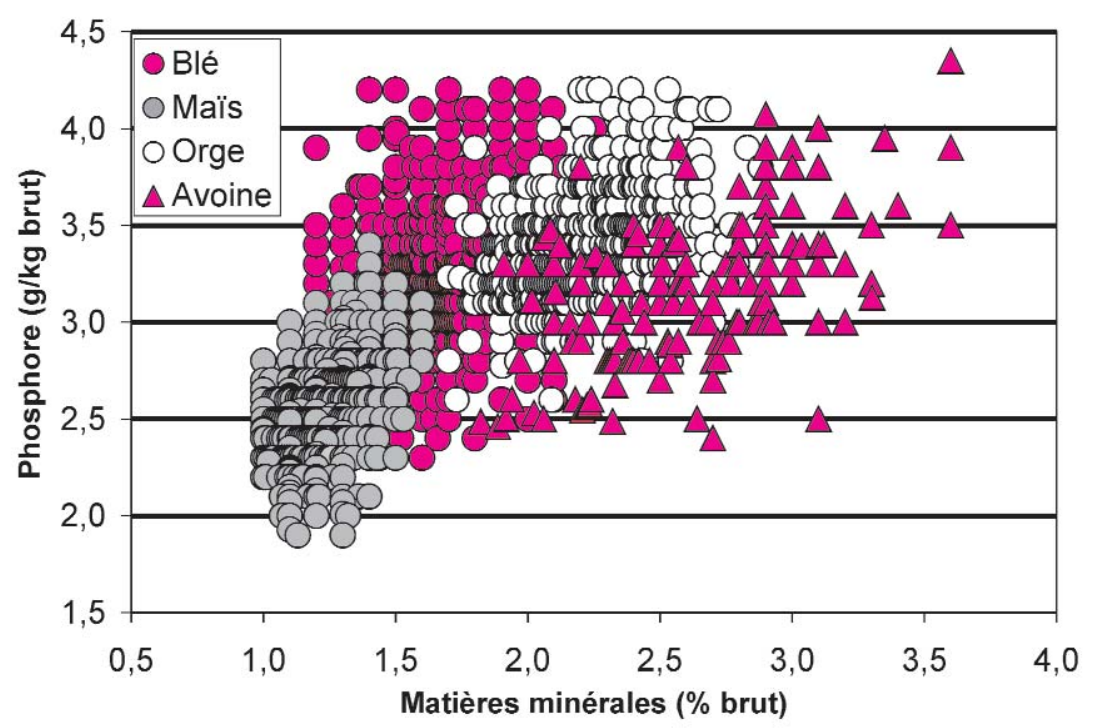

Figure 3b. Teneurs en phosphore total ( $g / k g$ brut) du blé et des issues de blé.

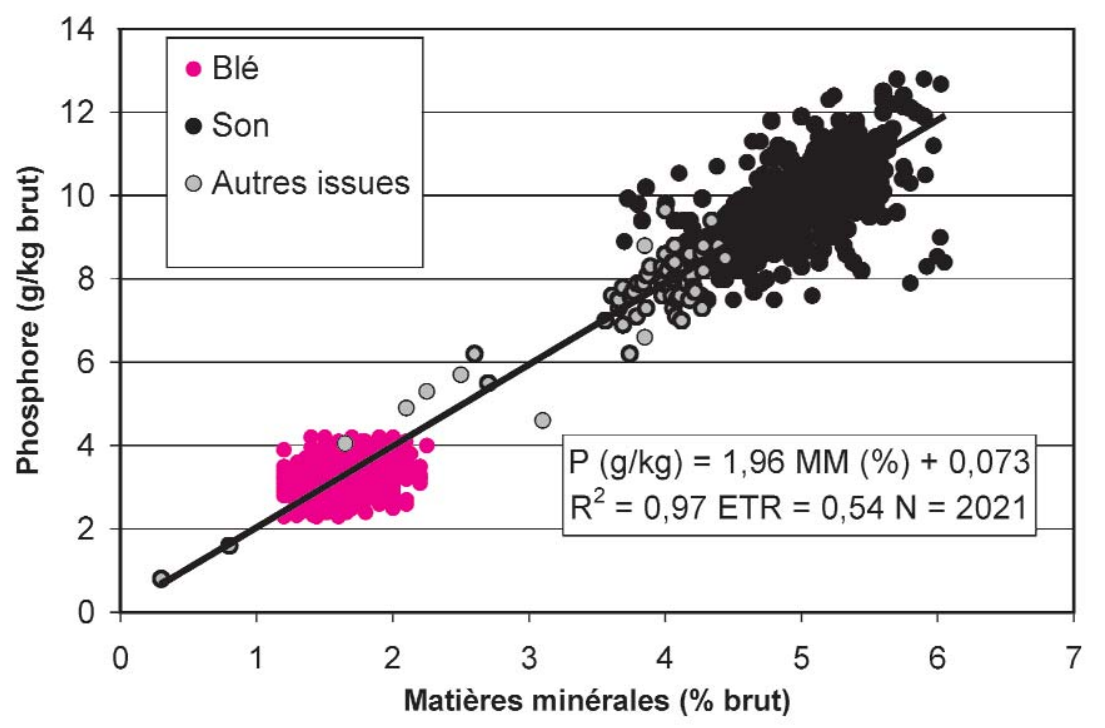

Figure 4. Teneurs en phosphore total ( $g / k g$ brut) des graines oléo protéagineuses.

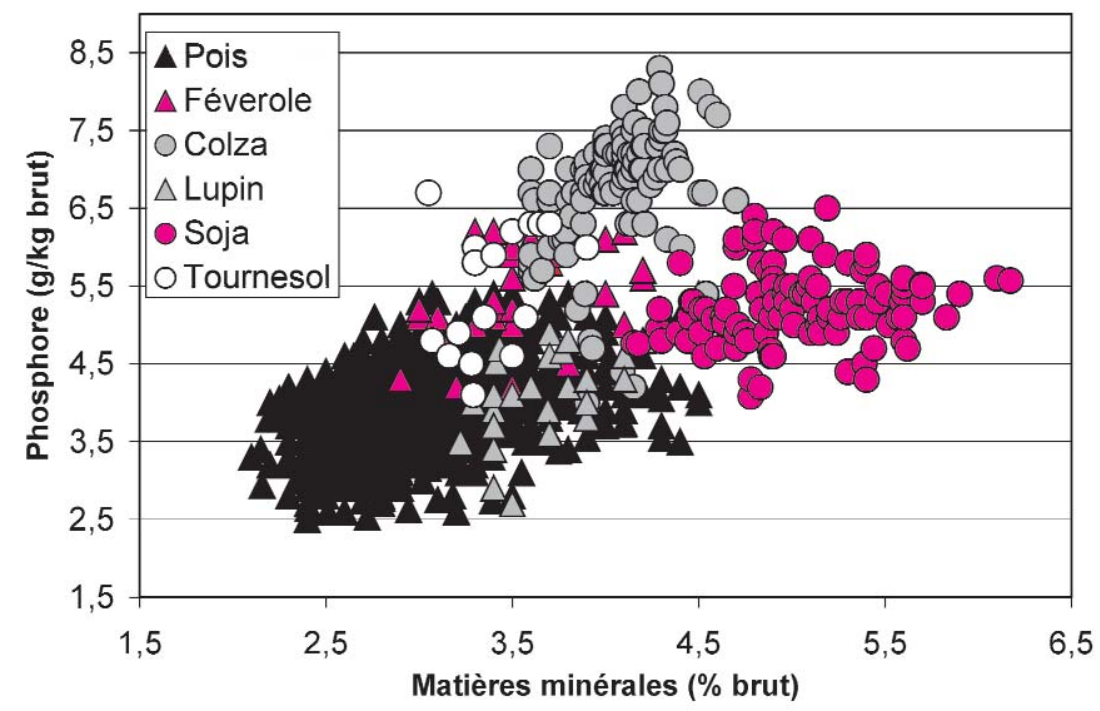

phosphore total assez voisines (10$11 \mathrm{~g} / \mathrm{kg})$, la variabilité de la teneur en phosphore du tourteau de tournesol est très supérieure à celle du tourteau de colza (écart-type de $1,6 \mathrm{~g} / \mathrm{kg}$ contre $0,9)$. En revanche, et bien que la plage de variation des matières minérales soit aussi étendue pour le tourteau de soja que pour le tourteau de tournesol, la variabilité du phosphore est faible pour le soja (écart-type de $0,5 \mathrm{~g} / \mathrm{kg}$ ).

La teneur en phosphore ne varie pas de la même façon en fonction des matières minérales pour le soja et son tourteau et pour les deux autres graines oléagineuses : $10 \mathrm{~g} / \mathrm{kg}$ de matières minérales correspondent à $1 \mathrm{~g} / \mathrm{kg}$ de phosphore pour le soja, et à 1,6 g/kg pour le colza et le tournesol (graines et tourteaux).

\section{4 / Autres produits végétaux}

Les produits issus d'autres parties de la plante que la graine (parties aériennes, fruits, racines) ont souvent des teneurs en phosphore faibles tant en valeur absolue (moins de 3,5 g/kg) qu'en valeur relative (ratio P/MM inférieur à $5 \%$ ). Il n'y a alors pas toujours de corrélation entre la teneur en matières minérales et la teneur en phosphore. Dans le cas des pulpes de betterave et des luzernes déshydratées (figure 6), on remarque que la teneur en phosphore varie peu (écart-type de $0,3 \mathrm{~g} / \mathrm{kg}$ ) en dépit d'une plage de variation très importante des teneurs en matières minérales. On peut faire l'hypothèse, au moins dans le cas des pulpes de betterave, que la variation des matières minérales est essentiellement due à un apport externe (terre adhérant aux racines lors de la récolte) pauvre en phosphore.

\section{5 / Farines de poisson}

La farine de poisson (figure 7) présente une grande variabilité de la teneur en matières minérales du fait des différentes matières premières traitées : espèce de poisson, origine géographique, type de produit (déchets de conserverie, poissons entiers). La teneur en phosphore varie de 16 à 40 g/kg (écart-type de l'ordre de $4 \mathrm{~g} / \mathrm{kg}$ ) et est fortement liée à la teneur en matières minérales avec un ratio $\mathrm{P} / \mathrm{MM}$ de l'ordre de $16 \%$.

\section{6 / Phosphates}

Les phosphates les plus courants en alimentation animale sont le phosphate bicalcique, le phosphate monocalcique et le phosphate monobicalcique (qui est 
Figure 5. Teneurs en phosphore total ( $\mathrm{g} / \mathrm{kg}$ brut) des tourteaux de colza, soja et tournesol.

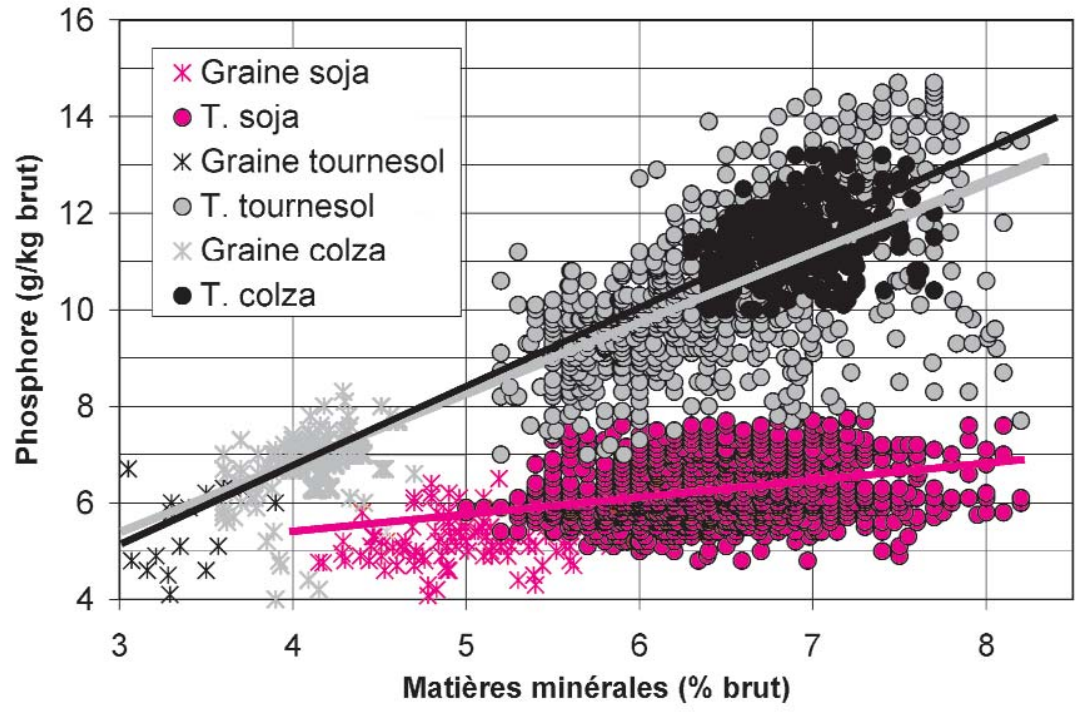

Figure 6. Teneurs en phosphore total ( $\mathrm{g} / \mathrm{kg}$ brut) de la pulpe de betterave, de la pulpe d'agrumes et de la luzerne déshydratée.

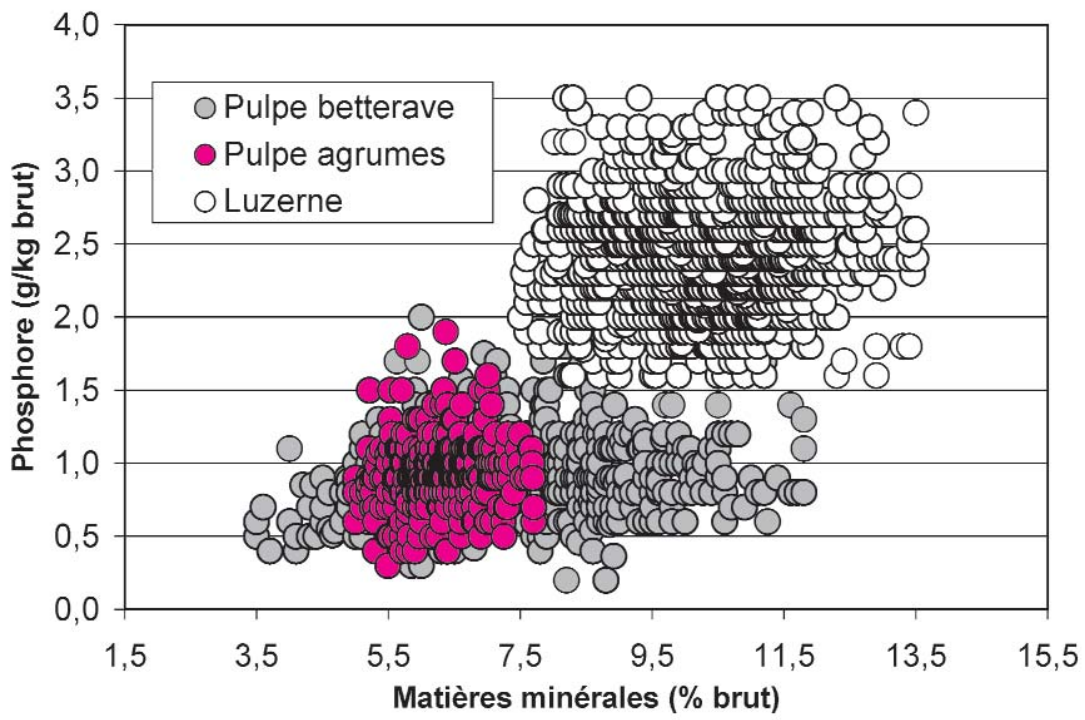

Figure 7. Teneurs en phosphore total ( $\mathrm{g} / \mathrm{kg}$ brut) des farines de poisson.

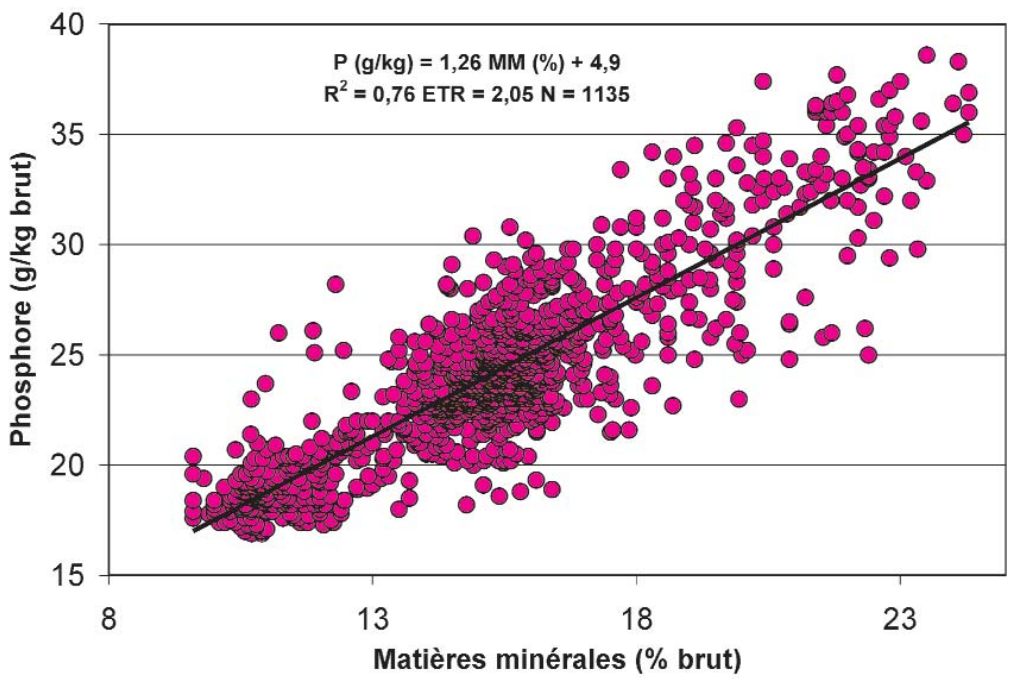

composé des deux précédents en proportions variables). Certains de ces phosphates sont assortis de garanties commerciales sur les teneurs en phosphore et calcium. En ce qui concerne le phosphore, on peut regrouper les phosphates de calcium en deux grandes familles : les phosphates bicalciques, qui contiennent environ $180 \mathrm{~g} / \mathrm{kg}$ de phosphore, et les mono et monobicalciques, qui en contiennent 210-220 g/kg. Les plages de variation observées sont importantes (de 150 à $210 \mathrm{~g} / \mathrm{kg}$ pour un bicalcique), avec des écarts-types de l'ordre de $10 \mathrm{~g} / \mathrm{kg}$ (soit un $\mathrm{CV}$ relativement faible de 4-6\%) (figure 8).

\section{2 / Teneurs en phosphore phytique}

Le phosphore phytique est la forme de stockage du phosphore organique des plantes, présent sous forme d'un complexe avec l'acide phytique. Ce phosphore n'est que très peu disponible pour les monogastriques qui ne sécrètent pas de phytases permettant d'hydrolyser les groupements phosphates de l'acide phytique et doivent utiliser les phytases des microorganismes du tube digestif, dont l'efficacité peut être faible.

Il est donc nécessaire de connaître la teneur en phosphore phytique pour bien évaluer la disponibilité du phosphore : dans le cas du porc, le phosphore phytique entre notamment dans des équations linéaires multiples de prédiction de la digestibilité du phosphore des matières premières (Weremko et al 1997).

Pour la majorité des matières premières (figure 9), le phosphore phytique constitue de 50 à $80 \%$ du phosphore total (Pointillart, 1994). Quelques produits végétaux (manioc, certains concentrés protéiques) ont des teneurs en phosphore phytique inférieures à $25 \%$ du phosphore total et les produits animaux n'en contiennent pas.

Ces ratios sont cependant très variables pour une même espèce. Dans le cas du blé et du pois, par exemple, on constate des plages de variation très importantes autour de la moyenne (30-95\% pour le blé et 15-90 \% pour le pois) (figure 10).

\section{3 / Activité phytasique}

Les phytases peuvent être présentes dans certains végétaux (phytases végé- 
Figure 8. Teneurs en phosphore total (\% brut) des grands types de phosphates de calcium.

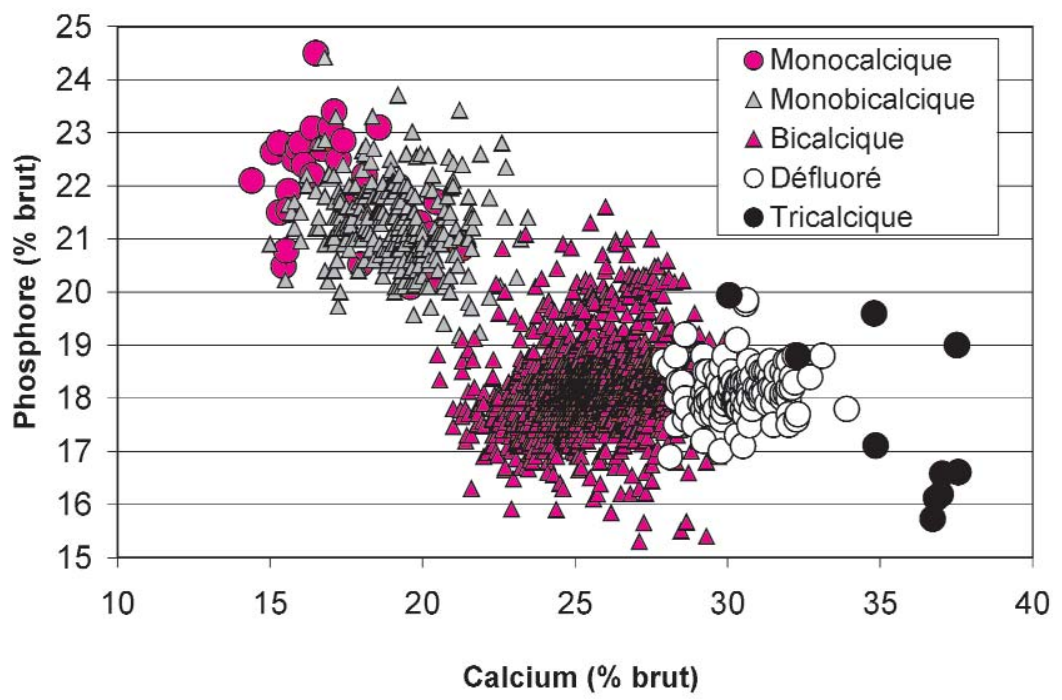

Figure 9. Teneurs en phosphore phytique ( $\mathrm{g} / \mathrm{kg}$ brut) des principales familles de matières premières (INRA-AFZ 2004).

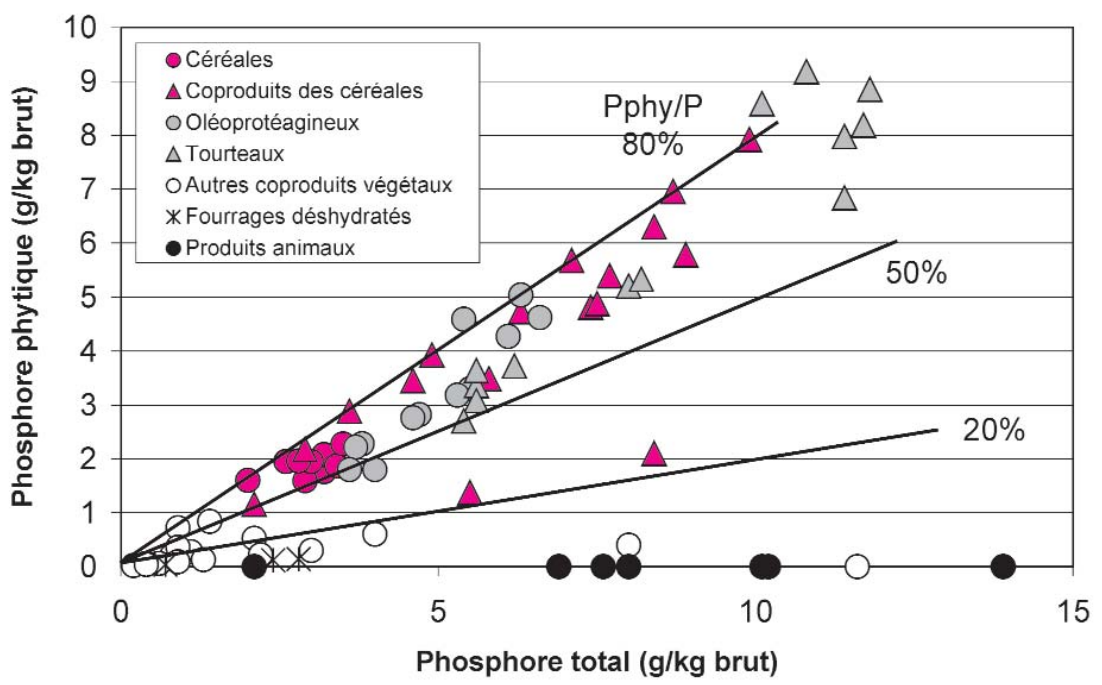

Figure 10. Variabilité du ratio $P$ phytique / $P$ total pour le blé, la féverole, l'orge et le pois.

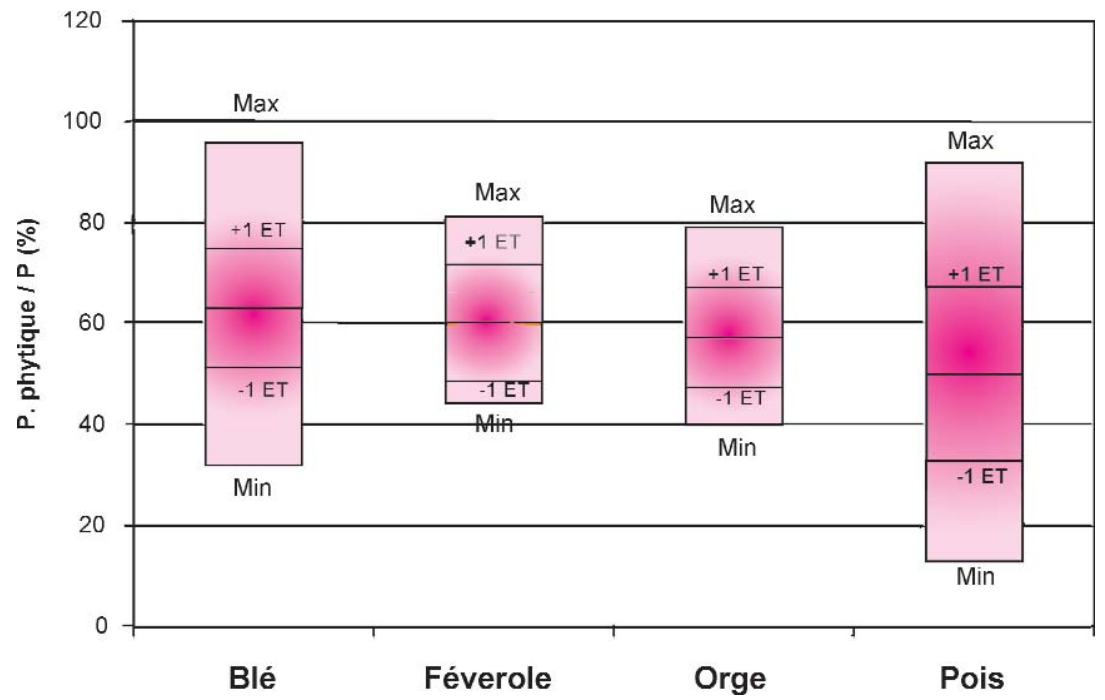

tales ou endogènes) mais peuvent aussi être produites par des microorganismes (phytases microbiennes ou exogènes). Leur action consiste à libérer le phosphore de l'acide phytique, le rendant assimilable par l'animal, notamment chez les monogastriques. Une unité de phytase correspond à la libération d'une umol par minute de phosphore inorganique à partir d'une solution de phytate de sodium, à une température et à un $\mathrm{pH}$ donné. Les phytases végétales sont présentes de façon significative dans le seigle (plus de $5000 \mathrm{U} / \mathrm{kg}$ ), les issues de blé, le triticale, l'orge et le blé, où elles sont considérées comme de bons prédicteurs de la digestibilité de ces matières premières chez le porc (Skiba et al 2004, Pointillart 1993) (figure 11).

L'activité phytasique endogène est très variable. Pour le blé tendre (figure 12 ), on constate des valeurs allant de 250 à plus de $1000 \mathrm{U} / \mathrm{kg}$ avec un CV de l'ordre de $30 \%$, soit une variabilité trois fois plus élevée que celle concernant la teneur en phosphore ou en phosphore phytique.

\section{4 / Facteurs de variation}

\section{1 / Effets variétaux et cultu- raux}

Arvalis - Institut du végétal a étudié sur la récolte 2001, dans le cadre d'un programme ACTA, les effets du site de culture (6 sites) et de la variété (7 cultivars) sur les teneurs en phosphore et phosphore phytique et sur l'activité phytasique du blé tendre $(n=37)$.

L'effet lieu est significatif sur les trois variables (figures 13 et 14). En revanche, l'effet variété n'est significatif que sur l'activité phytasique en accord avec les résultats de Barrier-Guillot et al (1996). Par ailleurs, une relation globale (inter lieux et variétés) significative $\left(r^{2}=0,72\right)$ a été trouvée entre phosphore et phosphore phytique confirmant les résultats de Barrier-Guillot et al (1996). Dans le cadre de ce programme, une étude a également été conduite sur 5 variétés de triticale cultivées dans 5 lieux. On observe alors un effet significatif du lieu sur les teneurs en $\mathrm{P}$, $\mathrm{P}$ phytique et activité phytasique mais aucun effet variétal.

Oury et al (1998) ont étudié la teneur en phosphore et l'activité phytasique du blé sur deux sites, EstréesMons ( $n=60$, 30 variétés x 2 types de fertilisation azotée) et Clermont- 
Figure 11. Activité phytasique des matières premières (U/kg brut) (INRA-AFZ 2004).

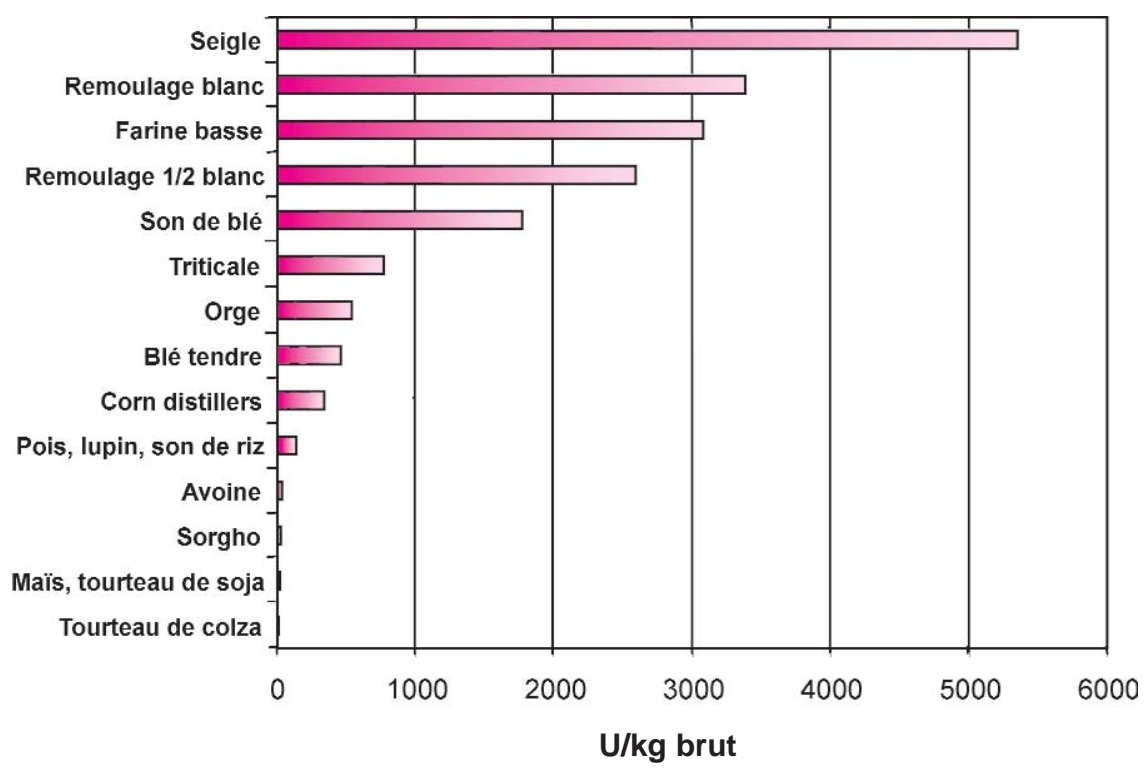

Figure 12. Variabilité de l'activité phytasique du blé tendre (U/kg brut).

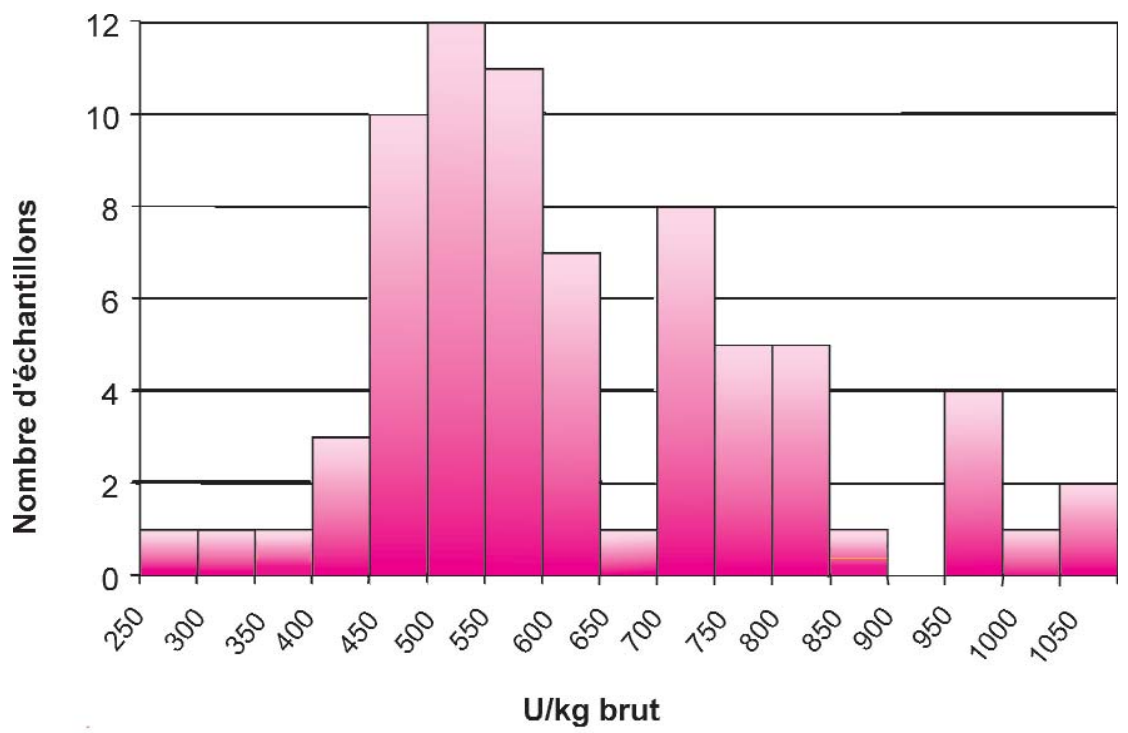

Ferrand $(\mathrm{n}=64,16$ variétés $\mathrm{x} 2$ dates de semis $\mathrm{x} 2$ types de fertilisation azotée). Leurs conclusions sont les suivantes. Il existe un effet positif de la fertilisation azotée sur l'activité phytasique sur un site (Clermont-Ferrand) et une augmentation de la teneur en phosphore lorsqu'il n'y a pas de fertilisation azotée (par rapport à une dose d'azote permettant un rendement de 90 quintaux par hectare) sur l'autre site. Ainsi, l'application tardive d'azote favoriserait le développement d'activités enzymatiques endogènes alors que l'absence d'azote diminuerait la compétition entre plantes pour le prélèvement de phosphore dans le sol d'où une augmentation de la teneur en phosphore du grain. Par ailleurs, les auteurs observent un effet très significatif du génotype sur l'activité phytasique et significatif sur le phosphore. Mais ils concluent également que le phosphore et l'activité phytasique sont des critères sous dépendance d'une interaction génotype $x$ environnement, cette dépendance induisant une forte variabilité des valeurs mesurées. Il en résulte que ces paramètres sont difficilement sélectionnables. Quant à la date de semis ou le rendement, ils n'ont pas d'effet sur la teneur en phosphore et l'activité phytasique. Enfin, comme Barrier-Guillot et al (1996) ils n'observent pas de relation entre la teneur en phosphore et l'activité phytasique.

Kim et al (2002) ont étudié en Australie l'effet de la variété, de l'année de récolte, de la zone de précipitation et du stockage sur la teneur en phosphore, la teneur en phosphore phytique et l'activité phytasique du blé $(\mathrm{n}=18$, 3 variétés $\mathrm{x} 2$ années de récolte $\mathrm{x} 3$ zones de

Figure 13. Effet du lieu et de la variété sur la teneur en phosphore et en phosphore phytique du blé (g/kg MS).

Effet Lieu

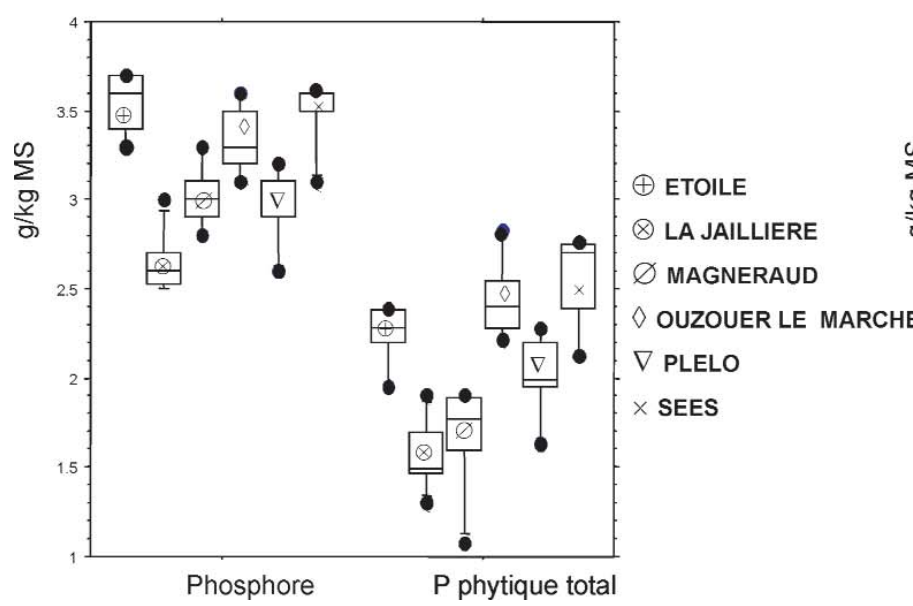

Effet Variété

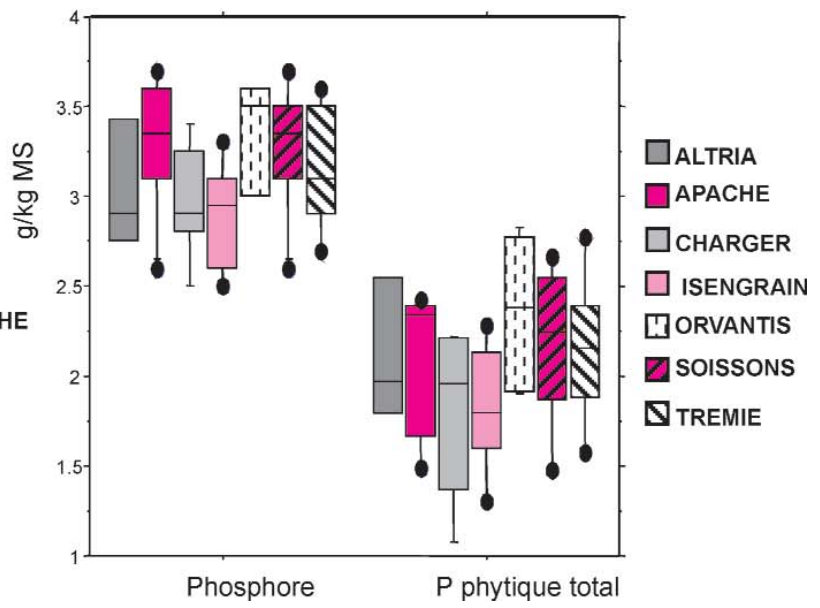


Figure 14. Effet du lieu et de la variété sur l'activité phytasique du blé (U/kg MS).

Effet Lieu

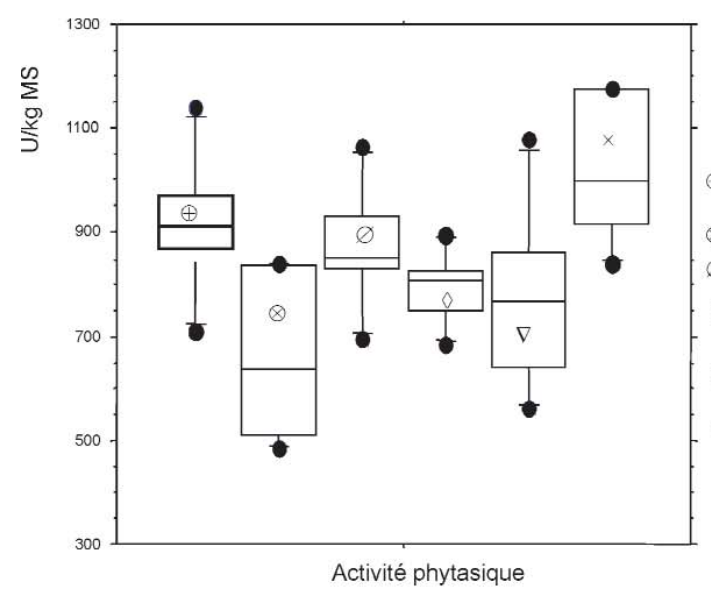

précipitation). Ils observent un effet significatif du lieu (zone de précipitation), $\mathrm{P}$ total et $\mathrm{P}$ phytique augmentant avec les précipitations ( $\mathrm{r}^{2}$ faibles mais significatifs). L'effet du génotype n'est significatif que sur l'activité phytasique. Ils mettent en évidence une relation significative entre $\mathrm{P}$ phytique et $\mathrm{P}$ total $\left(\mathrm{r}^{2}=0.95\right)$ comme Barrier-Guillot et al (1996). Par contre, aucun effet de l'année de récolte ni du stockage à température ambiante en grain pendant 6 mois n'est observé sur les différents critères étudiés. L'absence d'effet de la durée de stockage sur l'activité phytasique confirme les résultats de Nys et al (1996) dans le cas où les grains de blé sont conservés entiers. Par contre, si ces grains sont broyés, Nys et al (1996) observent une diminution linéaire de l'activité phytasique de la farine dans le temps (entre 0 et 8 mois).

Pour étudier l'effet de la fertilisation phospho-potassique, 22 échantillons de blé d'une variété (Aztec) cultivée sur des parcelles ayant reçu depuis 10 ans différents niveaux de fertilisation phosphatée associés à des rendements différents ont été collectés par Arvalis Institut du végétal dans le cadre du programme ACTA évoqué plus haut. On observe, d'une part, une réponse curvilinéaire du rendement au bilan fertilisation-exportation par la plante $(r=0,88)$ et, d'autre part, que la teneur en $\mathrm{P}$ total est corrélée $(r=0,84)$ à la Cp (teneur en $\mathrm{P}$ du sol extrait à l'eau). Barrier-Guillot et al (1996) avaient conclu de leur étude, sur 56 lots de blé, que les lots présentant les teneurs les plus faibles en phosphore étaient ceux recevant les doses de $\mathrm{P}_{2} \mathrm{O}_{5}$ les plus faibles. Par ailleurs, l'augmentation de la fertilisation $\mathrm{P}_{2} \mathrm{O}_{5}$ s'accompagnait d'une augmentation du pourcentage de phosphore présent sous forme phytique. Dans le cas d'une fertilisation phosphatée supérieure aux besoins de la plante, celle-ci stockerait donc l'excès de
Effet Variété

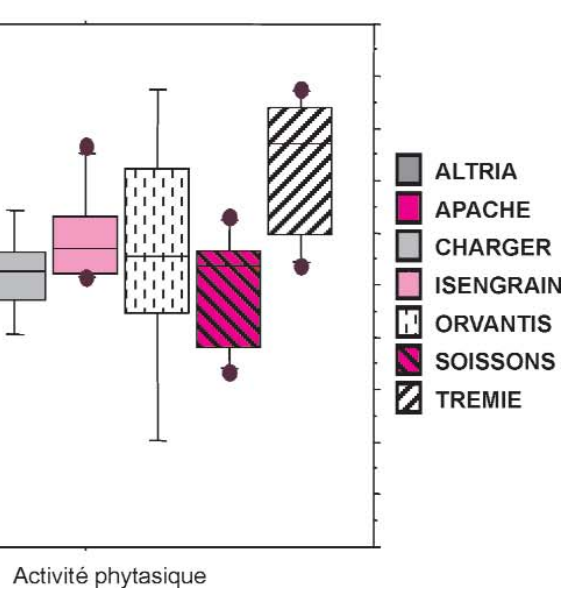

phosphore sous forme de phosphore phytique.

Arvalis - Institut du végétal a mesuré l'activité phytasique et le rapport phosphore phytique sur phosphore total sur 5 variétés d'orge d'hiver et 3 variétés d'orge de printemps des récoltes 2000 et 2002 (figure 15). Les variétés d'hiver ont une activité phytasique moindre que les variétés de printemps, et présentent des ratios $\mathrm{P}$ phytique / $\mathrm{P}$ total supérieurs en raison d'une teneur inférieure en $\mathrm{P}$ phytique dans les variétés de printemps. De telles observations n'ont, à notre connaissance, pas été rapportées par d'autres auteurs et mériteraient des investigations complémentaires.

Enfin, concernant le maïs, 10 échantillons (2 humidités $\times 2$ modes de conservation, 3 précocités $\mathrm{x}$ irrigation ou non) ont été collectés lors de la récolte 2001 par Arvalis - Institut du

Figure 15. Effet de la variété sur le ratio phosphore phytique / phosphore total (\%) et l'activité phytasique de l'orge (g/kg MS).

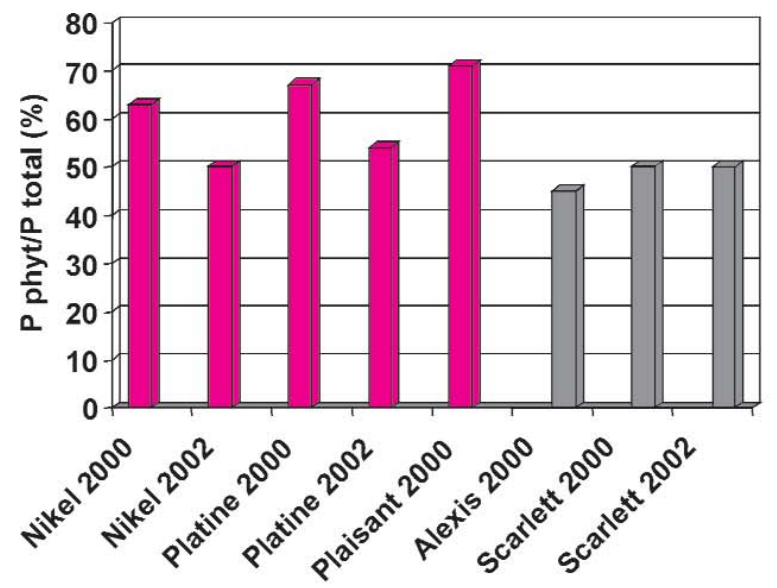

Hiver $\square$ Printemps

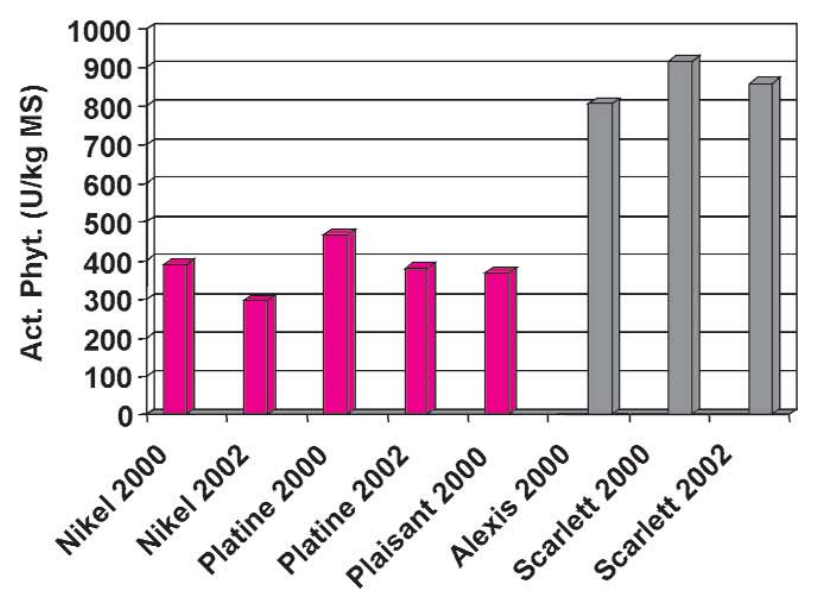

$\square$ Hiver $\square$ Printemps 
Figure 16. Effet de la température de granulation sur le ratio phosphore phytique / phosphore total (\%) et l'activité phytasique du blé ( $g / k g ~ M S)$.

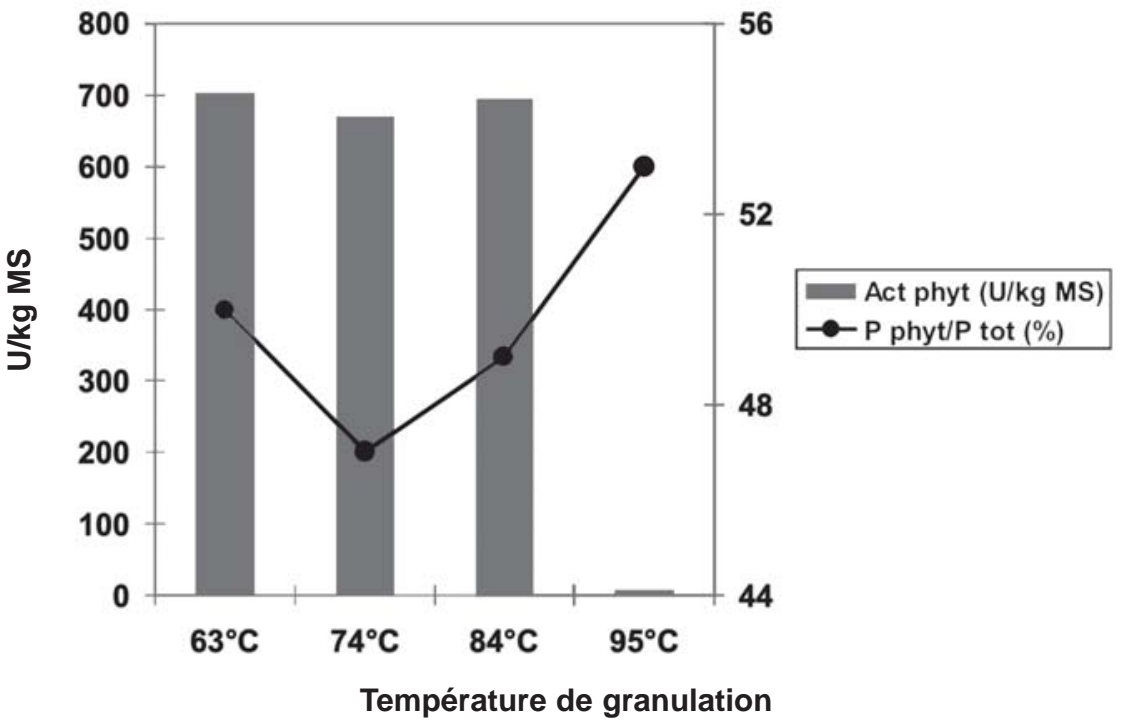

végétal dans le cadre du programme ACTA déjà évoqué, de façon à pouvoir étudier l'influence de la précocité variétale, de l'humidité à la récolte, de l'irrigation et du mode de conservation (sec vs humide entier inerté). Aucun des facteurs étudiés n'a eu d'effet sur les teneurs en phosphore total, phytique et activité phytasique.

On peut donc conclure de ces différentes études que c'est surtout le lieu de culture, à travers la fertilisation phosphatée voire azotée ou les précipitations, qui peut faire varier la teneur en phosphore ou en phosphore phytique des céréales (coefficient de variation d'environ $10 \%)$. De plus, il existe souvent une relation linéaire positive entre les deux critères. L'activité phytasique endogène est beaucoup plus variable (CV de 30 \%). Contrairement aux deux critères précédents, elle peut être sous détermi- nisme génétique mais souvent en interaction avec le lieu de culture, ce qui rend ce critère difficilement sélectionnable. Elle n'est pas reliée à la teneur en phosphore ou en phosphore phytique.

\section{2 / Effets de la technologie}

Un essai conduit par Arvalis - Institut du végétal en 2003 a permis d'observer l'effet de la température de granulation sur un aliment à base de blé $(97 \%$ de blé + AMV + carbonate, filière 2,5 x 35 ou 2,5 x 50 avec ou sans vapeur). La phytase endogène reste stable jusqu'à $85^{\circ} \mathrm{C}$ puis devient inactive à $95^{\circ} \mathrm{C}$, tandis que le ratio $\mathrm{P}$ phytique / $\mathrm{P}$ total augmente avec la température (figure 16).

Une autre étude conduite par ArvalisInstitut du végétal et INZO ${ }^{\circ}$ a étudié l'effet du traitement de thermisation des farines ( $85^{\circ} \mathrm{C}$ pendant 3 minutes) sur le ratio $\mathrm{P}$ phytique / $\mathrm{P}$ total (\%) et l'activité phytasique (g/kg MS) de plusieurs matières premières courantes (maïs, tourteau de soja, blé, orge et pois). L'effet est toujours négatif et significatif sur l'activité phytasique mais moins marqué, voire non significatif, sur le ratio $\mathrm{P}$ phytique / $\mathrm{P}$ total (figure 17 ).

D'autres auteurs ont fait des observations similaires quant à l'effet de la température sur l'activité phytasique endogène : inactivation partielle si $10 \mathrm{~min}$ à $80^{\circ} \mathrm{C}$ (Peers, 1953), inactivation si température de granulation supérieure à $80^{\circ} \mathrm{C}$ (Jong-bloed et Kemme, 1990). La synthèse des résultats présentés par Nys et al (1996) montre qu'une forte perte d'activité phytasique endogène intervient autour de $80-85^{\circ} \mathrm{C}$, les résultats étant variables suivant les études et donc sans doute selon les conditions de granulation. En revanche, ni Ranhotra et Loewe (1975) sur du blé, ni Ma et Shan (2002) sur du blé, du triticale et du seigle n'observent de diminution significative de l'activité phytasique endogène sous l'effet de la chaleur (y compris à $100^{\circ} \mathrm{C}$ pendant $1 \mathrm{~h}$ avec chaleur sèche).

Ces résultats montrent donc que, au moins sous certaines conditions, les phytases végétales peuvent être sensibles à la chaleur, comme cela a été montré également pour les phytases exogènes (Nys et al 1996), et qu'il convient d’évaluer spécifiquement pour chaque atelier de fabrication l'effet de la technologie sur l’activité phytasique.

\section{3 / Effets de la méthode de dosage sur l'activité phytasique}

Il n'existe pas de méthode normalisée de dosage de l'activité phytasique.

Figure 17. Effet du traitement thermique sur l'activité phytasique (U/kg MS) et le ratio phosphore phytique / phosphore total (\%) du maïs, du tourteau de soja, du blé, de l'orge et du pois.
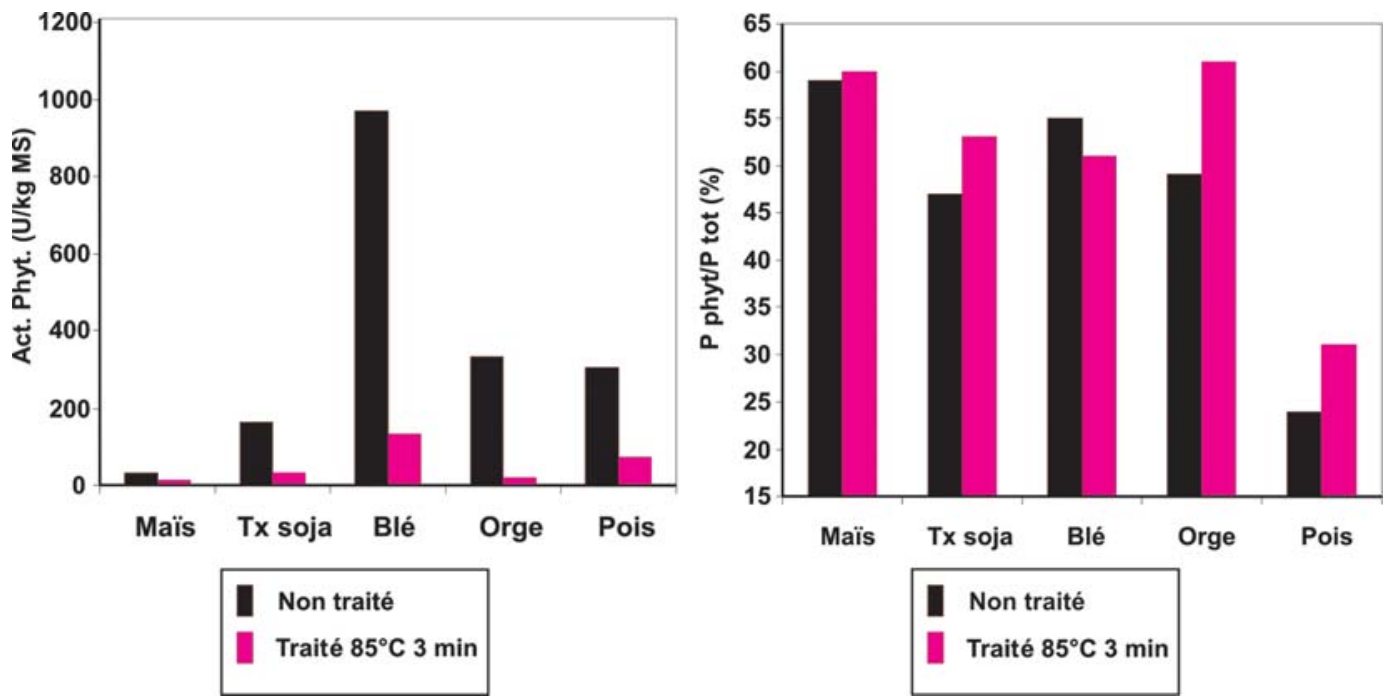
Figure 18. Dosage de l'activité phytasique de 5 lots de triticale et 5 lots de blés (conservés à $4^{\circ} \mathrm{C}$ ) à 10 ans d'intervalle par le même laboratoire.

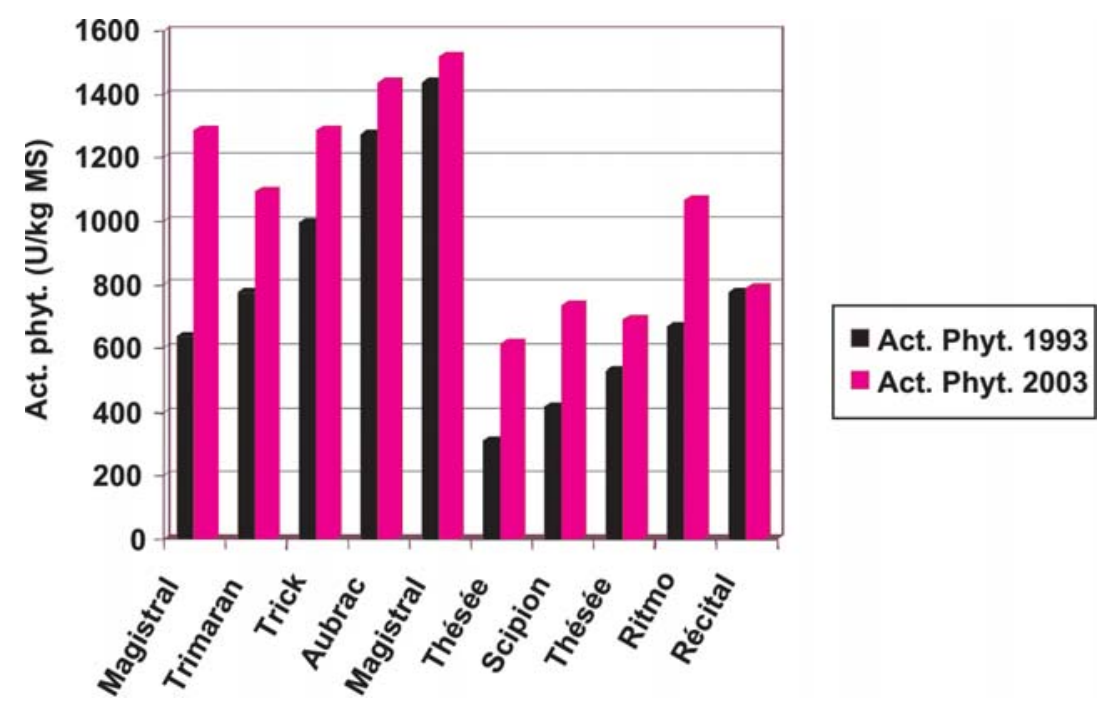

Chaque laboratoire a donc développé sa propre méthode, celle-ci ayant pu par ailleurs évoluer dans le temps. Arvalis Institut du végétal a ainsi comparé les résultats des mesures obtenues sur 5 blés figurant dans la publication de BarrierGuillot et al (1996) et 5 triticales avec de nouvelles analyses effectuées en 2003 dans le même laboratoire sur les grains conservés 10 ans à $4^{\circ} \mathrm{C}$ (figure 18). Même si la hiérarchie reste globalement comparable entre variétés de blés ou de triticales et que les valeurs obtenues sur triticales sont toujours supérieures aux valeurs obtenues sur les blés, l'augmentation des valeurs d'activité phytasique entre 1993 et 2003 va de 2 à $100 \%$, ce qui est considérable. Par ailleurs, des comparaisons de mesures effectuées entre Arvalis - Institut du végétal et un autre laboratoire ont montré, avant rapprochement des procédures analytiques, des différences aussi importantes pour un même lot de blé. Il convient donc de considérer la comparaison de résultats de mesures provenant de dates très différentes ou de laboratoires différents avec la plus grande précaution.

\section{Conclusions}

Les teneurs en phosphore et phosphore phytique des matières pre- mières peuvent être très variables. Pour les grains, les graines et leurs coproduits ainsi que les produits animaux, il existe cependant une certaine homogénéité dans un même type biologique (espèce végétale notamment), ce qui permet souvent d'établir une corrélation avec la teneur en matières minérales. Pour les produits issus d'autres parties de la plante (racines, feuilles...), il est plus difficile d'établir des corrélations utiles. Les mesures de phosphore et phosphore phytique présentent des CV de $10 \%$ environ alors que l'activité phytasique présente des $\mathrm{CV}$ allant jusqu'à $30 \%$.

Les conditions pédo-climatiques (lieu, précipitation, voire fertilisation) influencent directement les teneurs en phosphore et en phosphore phytique ainsi que l'activité phytasique. Cette dernière dépend aussi de facteurs génétiques, des traitements technologiques et des méthodes de dosage. L'existence d'interactions entre génotype et environnement rend difficile la sélection génétique sur ces critères.

De façon générale, la prédiction des teneurs en phosphore et surtout de l'activité phytasique n'est pas aisée, et les mesures de laboratoire sont nécessaires.

\section{Références}

Barrier-Guillot B., Casado P., Maupetit P., Jondreville C., Gatel F., 1996. Wheat phosphorus availability : 1-In vitro study ; Factors affecting endogenous phytasic activity and phytic phosphorus content. J. Sci. Food Agric., 70, 6268.

INRA-AFZ, 2004. Tables de composition et de valeur nutritive des matières premières destinées aux animaux d'élevage : porcs, volailles, bovins, ovins, caprins, lapins, chevaux, poissons. D. Sauvant, J.-M. Perez, G. Tran (eds), $2^{\mathrm{e}}$ édition révisée, INRA, Paris, 301p.

Jongbloed A.W., Kemme P.A., 1990. Effect of pelleting mixed feeds on phytase activity and the apparent absorbability of phosphorus and calcium in pigs. Anim. Feed Sci. Technol., 28, 233242.

Kim J.C., Mullan B.P., Selle P.H., Pluske J.R., 2002. Levels of total phosphorus, phytatephosphorus, and phytase activity in three varieties of Western Australian wheats in response to growing region, growing season, and storage. Austr. J. Agric. Res., 53(12), 13611366.

Oury F.X., Carré B., Pluchard P., Berard P., Nys Y., Leclercq B., 1998. Genetic variability and stability of poultry feeding related characters in wheat, in relation to environmental variation. Agronomie, 18(2), 139-150.

Ma X., Shan A., 2002. Effect of germination and heating on phytase activity in cereal seeds. Asian-Austral. J. of Anim. Sci., 15(7), 1036-1039.

Nys Y., Frapin D, Pointillart A., 1996. Occurrence of phytase in plants, animals and microorganisms. In: Phytase in animal nutrition and waste management. M.B. Coelho and E.T. Kornegay (eds), BASF, 213-236, USA.

Peers F.G., 1953. The phytase of wheat. Biochem. J., 53, 102-110.
Pointillart A., 1993. Importance of phytates and cereal phytases in the feeding of pigs. Proc $1^{\text {st }}$ Symp Enzymes Anim Nutr., Kartause Ittingen, Switzerland, 192-198.

Pointillart A., 1994. Phytates, phytases : leur importance dans l'alimentation des monogastriques. INRA Prod. Anim., 7, 29-39.

Ranhotra G.S., Loewe R.J., 1975. Effect of wheat phytase on dietary phytic acid. J. Food Sci., 40 (5), 940-942.

Skiba F., Callu P., Castaing J., Paboeuf F., Chauvel J., Jondreville C., 2004. Variabilité intra matière première de la digestibilité du phosphore des céréales et du pois chez le porc en croissance. Journ. Rech. Porcine Fr., 36, 9-16.

Weremko D., Fandrejewski H., Zebrowska T., Han, In K., Kim J.H., Cho W.T., 1997. Bioavailability of phosphorus in feeds of plant origin for pigs (A review). Asian-Aust. J. Anim. Sci., 6 (10), 551-566. 


\section{Résumé}

La valeur "phosphore» des aliments, dont la connaissance est nécessaire pour maîtriser les apports nutritionnels et les rejets dans l'environnement, est caractérisée par les teneurs en phosphore total et en phosphore phytique par l'activité phytasique endogène. Les teneurs en phosphore total varient fortement entre matières premières : de moins de $1 \mathrm{~g} / \mathrm{kg}$ (paille de blé) à plus de $210 \mathrm{~g} / \mathrm{kg}$ (certains phosphates). Le phosphore total représente en général moins de $20 \%$ des matières minérales et le ratio phosphore total / matières minérales (P/MM) est caractéristique d'une famille biologique. Ainsi, pour les céréales et les graines oléagineuses (2 à $8 \mathrm{~g} / \mathrm{kg}$ de phosphore total), l'extraction de l'amidon ou de l'huile conduit à une concentration en matières minérales avec un ratio $\mathrm{P} / \mathrm{MM}$ relativement constant pour une espèce donnée (20\% pour le blé, $10 \%$ pour le soja). Pour les produits végétaux autres que les grains et graines et leurs coproduits, les teneurs en phosphore total sont plus faibles (moins de $3 \mathrm{~g} / \mathrm{kg}$ ) et moins corrélées aux teneurs en matières minérales.

Le phosphore phytique constitue en général de 50 à $80 \%$ du phosphore total des matières premières végétales, avec une grande variabilité intra-espèce ( 30 à $90 \%$ pour le blé). Les phytases végétales sont présentes de façon significative dans le seigle (plus de $5000 \mathrm{U} / \mathrm{kg}$ ), les issues de blé, le triticale, l'orge et le blé, avec là encore une grande variabilité intra-espèce (de 250 à 1000 U/kg pour le blé).

Différentes études montrent que le principal facteur de variation de la teneur en phosphore ou en phosphore phytique des céréales est le lieu de culture, à travers la fertilisation phosphatée et azotée ou le climat. Il existe souvent une relation linéaire reliant les deux critères. L'activité phytasique endogène semble surtout déterminée génétiquement mais en interaction avec le lieu de culture, ce qui rend ce critère difficilement sélectionnable. Elle n'est pas liée à la teneur en phosphore ou en phosphore phytique. Par ailleurs, les phytases végétales, comme les phytases exogènes, sont sensibles à la chaleur et il convient d'évaluer spécifiquement pour chaque atelier de fabrication l'effet de la technologie sur l'activité phytasique. La mesure de cette activité est enfin fortement sujette à des variations inter-laboratoires. De façon générale, la prédiction des teneurs en phosphore et surtout de l'activité phytasique n'est pas aisée, et les mesures de laboratoire sont souvent nécessaires.

\section{Abstract}

Inter and intra feed material variability of the contents in total and phytic phosphorus and of phytase activity

Proper management of phosphorus nutrition and excretion requires a good knowledge of total phosphorus content, phytic phosphorus content and endogenous phytase activity of the feed ingredients. Total phosphorus content varies greatly between feed materials, from less than $1 \mathrm{~g} / \mathrm{kg}$ (wheat straw) to more than $210 \mathrm{~g} / \mathrm{kg}$ (some phosphates). Total phosphorus usually makes up about $20 \%$ of the mineral matter and the phosphorus / mineral matter ratio (P/MM) depends on the biological family of the feed material. For cereal grains and oilseeds (2 to $8 \mathrm{~g} / \mathrm{kg}$ of total $\mathrm{P}$ ), the removal of starch or oil results in a concentration of mineral matter in the by-product while P/MM remains fairly stable for a given species ( $20 \%$ for wheat, $10 \%$ for soybean). Plant feed materials other than seeds, grains and their by-products tend to have lower total phosphorus contents (less than $3 \mathrm{~g} / \mathrm{kg}$ ) that are less correlated with mineral matter content.

Phytic phosphorus makes up about 50 to $80 \%$ of the total phosphorus of plant feed materials, with a high intra-species variability (30-90\% for wheat). Plant phytases are significantly present in rye (more than $5000 \mathrm{U} / \mathrm{kg}$ ), wheat milling by-products, triticale, barley and wheat. Intra-species variability is also high (250-1000 U/kg for wheat).

Several studies have shown that the main factor of variation for the content in total and phytic phosphorus of cereals is the growing location (P or $\mathbf{N}$ fertilization, climate). Both types of phosphorus are also often linearly related. Endogenous phytase activity seems to be primarily determined by genetics, but, since it is also related to the growing location, it does not seem to be usable for genetic selection. It is not related to total or phytic phosphorus content. Plant phytases are heat sensitive and the effect of technology on phytase activity should be monitored at each stage of the feed manufacturing process. In addition, phytase activity is subject to large inter-laboratory variability. In any case, predicting phosphorus content and phytase activity is not easy and laboratory analysis is often required.

TRAN G., SKIBA F. 2005. Variabilité inter et intra matière première de la teneur en phosphore total et phytique et de l'activité phytasique. INRA Prod. Anim., 18, 159-168. 